\title{
SEX HORMONE PREPARATIONS AND RETINAL VEIN OCCLUSION
}

\author{
J. F. KIRWAN ${ }^{1}$, M. D. TSALOUMAS ${ }^{2}$, H. VINALL ${ }^{3}$, P. PRIOR ${ }^{3}$, E. E. KRITZINGER ${ }^{2}$ and \\ P. M. DODSON ${ }^{3}$ \\ Birmingham and Kingston upon Thames
}

\begin{abstract}
SUMMARY
Retinal vein occlusion (RVO) is most commonly seen in middle-aged or older patients and is associated with underlying cardiovascular risk factors. It is much less common in younger patients. Use of the oral contraceptive pill (OCP) is known to be a risk factor for cardiovascular and cerebrovascular disease. There have previously been a few isolated case reports of patients sustaining an RVO whilst taking the OCP. The aim of this study was to investigate patients sustaining an RVO whilst taking the OCP or hormone replacement therapy (HRT). From a large series of 588 patients, we found 11 with an RVO associated with sex hormone preparations. Of these, 6 had taken the OCP and 5 were HRT users. From this large group there were only 9 female patients aged under 35 years who sustained an RVO. Of these, 6 were associated with use of the OCP. All patients were investigated for recognised medical risk factors for RVO. None of these factors were identified in the patients who had used the OCP. Of the patients taking HRT, 4 of the 5 had other potential risk factors. From our large series, the prevalence of RVO in female patients under 35 years taking the OCP was $66 \%$. There is a 30\% uptake of the ОCP in the general population. These data support the view that $R V O$ is a contraindication to the use of the OCP. Additionally, it would appear (albeit from limited data) that patients who sustain RVO may continue with their HRT, as HRT is not a major single risk factor for RVO.
\end{abstract}

Retinal vein occlusion (RVO) is an important cause of visual loss, particularly in middle age. ${ }^{1}$ The exact aetiology of RVO is unclear. Histological studies by Klein $^{2}$ and Rabinowicz $z^{3}$ show two different principal factors in the mechanism of occlusion: primary endothelial degeneration and swelling, secondary

From: ${ }^{1}$ Royal Eye Unit, Kingston Hospital, Kingston upon Thames; ${ }^{2}$ Birmingham and Midland Eye Hospital, Birmingham;

${ }^{3}$ Birmingham Heartlands Hospital, Birmingham, UK.

Correspondence to: P. M. Dodson, MD. FRCP, FRCOphth. Birmingham Heartlands Hospital, Bordesley Green East. Birmingham B9 5SS, UK. intramural formation of thrombus and, secondly, extreme 'phlebosclerosis' with secondary endothelial degeneration. Studies have shown abnormalities in patients with RVO that may predispose to both these mechanisms. These include an increased prevalence of hypertension, hyperlipidaemia, enhanced in vivo platelet activity and the possibility of increased inflammatory activity.

RVO does occur in younger patients, with hyperlipidaemia being a more predominant associated factor than hypertension. Additionally, in younger patients, inflammatory causes can be important. From a large series of patients with RVO, predisposing factors in these younger patients were assessed. A number of patients taking sex hormone preparations have been recorded. ${ }^{4}$

The aim of this study was to examine the risk factor profile in patients sustaining RVO whilst taking sex hormone preparations, to determine whether the oral contraceptive pill (OCP) or hormone replacement therapy (HRT) could be implicated as independent risk factors in the aetiology of RVO.

\section{METHOD}

Five hundred and eighty-eight patients presenting after 1982 with RVO were reviewed in 1994. A number of younger patients were noted to be taking sex hormone preparations, notably the combined OCP and HRT. These patients were divided into two subgroups: (a) those taking the OCP $(n=6)$ and (b) those taking HRT $(n=5)$. From 1982, patients presenting to the Birmingham and Midland Eye hospital have been managed with combined ophthalmic and medical input. Patients were seen by a medical ophthalmologist (E.E.K.) for management of their RVO and complications. Diagnosis of RVO was made on clinical grounds, with fundus fluorescein angiography (FFA) if there was an element of doubt. Patients seen in the RVO clinic were also 
assessed for the presence of underlying medical conditions. Assessment consisted of consultation with a physician (P.M.D.) with full medical history and examination. The following baseline investigations were performed: full blood count, erythrocyte sedimentation rate (or plasma viscosity), serum fibrinogen, serum urea and electrolytes, blood cholesterol and triglyceride levels, serum immunoglobulins, plasma electrophoresis, electrocardiogram and chest radiography. Particular attention was paid to the presence of hypertension, hyperlipidaemia, smoking habits and alcohol consumption, the presence of other medical conditions, and drug therapy. Patients taking the OCP or HRT had their medication stopped, as in the 1980s this treatment was believed to be detrimental.

Definitions for the purpose of this study included that of the World Health Organization for hypertension (systolic $>160 \mathrm{mmHg}$, diastolic $>95 \mathrm{mmHg}$ on three occasions of if the patient was already taking antihypertensive drug therapy). Hyperlipidaemia was defined as fasting serum cholesterol $>6.5 \mathrm{mmol} /$ 1 and/or fasting serum triglyceride levels $>2.1 \mathrm{mmol} / 1$.

\section{RESULTS}

Clinical data obtained on patients taking the OCP are shown in Table I. Ages ranged from 24 to 33 years, with a mean of 27 years 9 months. All but 1 patient was taking the combined oestrogen/progestogen OCP (COCP); the other patient was taking the progestogen-only pill or 'mini-pill' (POP). Duration of use ranged from 2 to 14 years with a mean of 7 years 4 months. Two of the patients, indicated with an asterisk in Table I, had stopped using the OCP prior to the onset of RVO, the first 11 months and the second 5 months prior to that time.

None of the patients smoked, but two patients were ex-smokers. All but 1 patient consumed less than 16 units of alcohol weekly; the other (no. 3) consumed 28 units weekly. None of the patients was hypertensive, hyperlipidaemic, diabetic, or had any other identified risk factor for RVO. All other blood tests were normal.

Branch retinal vein occlusion (BRVO) was present in 2 patients; 4 had a central retinal vein occlusion
(CRVO). Two patients had a poor visual outcome, one with CRVO and one with BRVO. One patient (no. 5) had an earlier RVO, continued to take the $\mathrm{OCP}$, and then later had a more extensive recurrent RVO with a poor visual outcome.

The data obtained on patients taking HRT are shown in Table II. Ages ranged from 48 to 59 years with a mean of 53 years 6 months. Duration of use ranged from 1 month to 7 years. All were current users of HRT. All patients were taking HRT for relief of menopausal symptoms. Two patients took an oestrogen-only type preparation, the other 3 took a combined oestrogen/progestogen preparation.

Two patients were hypertensive, two patients were smokers, and 1 of these was also hypertensive, on treatment. One patient had a raised serum cholesterol of $7.2 \mathrm{mmol} / \mathrm{l}$, despite treatment at the time with clofibrate. All patients consumed less than 6 units of alcohol per week. One patient (no. 11) had a raised ESR, but plasma viscosity, C-reactive protein measurements and all other blood tests were normal. All patients except 1 had a good final visual acuity. Three patients had a BRVO and 2 had a CRVO.

\section{DISCUSSION}

The effects of the OCP and HRT on blood rheology and cardiovascular risk are different owing to the different dosages used. Discussion will therefore consider these two therapies separately.

The OCP has been shown to increase the risk of thromboembolism, as well as increasing risk for cardiovascular and cerebrovascular disease. ${ }^{5-7}$ This is the case even in patients with no other particular identifying risk factors. This effect is independent of other risk factors such as hypertension and smoking, although a combination of risk factors further increases the relative risk. ${ }^{8}$ Given the close similarity between risk factors for RVO and cardiovascular and cerebrovascular disease, it is therefore reasonable to suspect that the OCP may predispose towards RVO. However, given the scarceness of reports in the literature of RVO in patients taking the $\mathrm{OCP}$, and the relatively small numbers of $\mathrm{RVO}$ in younger patients, no study has provided evidence for a causal relationship.

Table I. Patient details of the oral contraceptive pill (OCP) group

\begin{tabular}{lcccccc}
\hline $\begin{array}{l}\text { Patient } \\
\text { no. }\end{array}$ & $\begin{array}{c}\text { Age } \\
\text { (years) }\end{array}$ & OCP type & $\begin{array}{c}\text { Duration of use } \\
\text { (years) }\end{array}$ & RVO type & Smoker & Visual acuity \\
\hline 1 & 24 & COCP & 8 & CRVO & Ex (3 years) & No \\
2 & 25 & POP & $*$ & BRVO & No & $6 / 9$ \\
3 & 28 & COCP & 2 & BRVO & No & $6 / 9$ \\
4 & 28 & COCP & 3 & CRVO & Ex (1 year) & $<6 / 60$ \\
5 & 30 & COCP & 14 & CRVO & No & $6 / 5$ \\
6 & 33 & COCP & $12 *$ & CRVO & . \\
\hline
\end{tabular}

No other risk factors were presented in these patients.

COCP, combined oral contraceptive pill; POP, progestogen-only pill; RVO, retinal vein occlusion; CRVO, central retinal vein occlusion; BRVO, branch retinal vein occlusion.

* These patients had stopped using the OCP prior to the onset of the RVO. 
Table II. Patient details of the hormone replacement therapy (HRT) group

\begin{tabular}{rccccccc}
\hline $\begin{array}{l}\text { Patient } \\
\text { no. }\end{array}$ & $\begin{array}{c}\text { Age } \\
\text { (years) }\end{array}$ & Duration of use & RVO type & Smoker & Hyperlipidaemia & Hypertension & Visual acuity \\
\hline 7 & 48 & N/K & BRVO & No & Yes & No & $6 / 36$ \\
8 & 50 & 18 months & BRVO & Yes & No & Yes & $6 / 6$ \\
9 & 55 & 2 years & CRVO & No & No & No & $6 / 6$ \\
10 & 56 & 1 month & CRVO & No & No & Yes & $6 / 6$ \\
11 & 59 & 7 years & BRVO & Yes & No & No & $6 / 6$ \\
\hline
\end{tabular}

When considering the epidemiological effects of OCP use, it is important to consider the changes in formulation, oestrogen dose and potencies over the last 30 years. Particularly significant is the reduction of the average oestrogen dose from $50 \mu \mathrm{g}$ to less than $30 \mu \mathrm{g}$ with modern 'low-dose' preparations. Some authors have suggested that with these newer preparations there may be no excess cardiovascular risk as noted in earlier studies. ${ }^{9}$ With regard to venous thromboembolism, and cerebrovascular disease, the evidence is less clear. The powerful synergistic effect of smoking combined with OCP use for cardiovascular risk has also been noted. ${ }^{10}$

In this large series of patients with RVO there were 9 women aged under 35 years with RVO. Nationally, the uptake of the OCP in patients in this age group (25-34 years) was $30 \%$ of the population during the period of the study. ${ }^{11}$ Thus by chance we would expect there to be 3 patients in this group taking the OCP. Instead, 6 of these 9 patients sustaining RVO were OCP users. This suggests a possible relationship, although numbers are too small for statistical interpretation. The inclusion of ex-users of the OCP in this study is important because although the excess cardiovascular risk is confined to current users, there is a past-use effect noted for cerebrovascular disease. ${ }^{12}$

Survey of the literature shows only a small number of patients sustaining RVO while taking the OCP. These had generally been reported as isolated occurrences, and to date the largest number of cases in any other series was 2 . However, anecdotal evidence suggests that this occurrence may make up a significant proportion of the young patients with RVO. Fong and Schatz ${ }^{13}$ commented that OCPassociated RVO is a 'very rare occurrence, and is probably associated with other contributing factors' a view not supported by our findings.

The case in which the OCP was not stopped is an interesting example. This patient had an RVO, continued with the OCP, and later went on to develop a further RVO with a poor visual outcome. The rate of re-occlusion is usually low, particularly in young patients. This suggests that cessation of the OCP is an important part of the management of these patients.

There are several possible mechanisms for the effect of the OCP on RVO risk. First, the OCP has been shown to increase coagulability by increasing levels of fibrinogen and factor VIIc. ${ }^{14.15}$ This effect is notably smaller in patients taking lower levels of oestrogen, but seems to be maintained for a period after cessation of the OCP. Hypertension is an important risk factor for RVO, although less so in young patients compared with an older cohort. It has been estimated that the OCP causes a rise in the diastolic blood pressure of $2 \mathrm{mmHg}$ and a rise of 5 $\mathrm{mmHg}$ in systolic blood pressure. ${ }^{16}$ This is a small but significant elevation. Such an increase would be expected to elevate the risk of cerebral infarction and coronary heart disease by 1.5 and 1.2 , respectively. The OCP is known to affect blood lipids, notably causing an increase in serum low density lipoprotein cholesterol (LDL) and a decrease in high density lipoprotein cholesterol (HDL) ${ }^{17.18}$ Hyperlipidaemia has been shown to be the major identified risk factor for RVO in young patients. ${ }^{19}$

In the HRT group of patients, an aetiological role for HRT is much less apparent. These patients are in an age group where RVO is considerably more common than in the younger group of patients. Seventy-one female patients between the ages of 40 and 60 years sustained an RVO, so these patients make up only $7 \%$ of this group, supporting the absence of an aetiological role for HRT. Additionally, 4 of 5 patients had other well-established risk factors for RVO. These data provide no evidence that HRT does predispose to RVO. Their importance is in the increasingly widespread uptake of HRT, and the reduction of some contraindications, such as its use in hypertensive patients. ${ }^{19}$ With a large and increasing proportion of the population using HRT, even a weak association may have significant effects on a susceptible population and it is important for any potential adverse effects to be reported. ${ }^{20}$

Unopposed oestrogens used in HRT seem to be protective against cardiovascular disease. However, their use is currently restricted to patients without uterine tissue. They have beneficial effects on lipoprotein metabolism, causing a reduction in total and LDL cholesterol and an increase in HDL cholesterol. $^{20}$ This effect on cardiovascular disease is, however, reduced by the inclusion of a progestogen, which appears to be the agent responsible for adverse effects on blood lipoproteins. ${ }^{21}$ Oestrogens themselves may, however, have an adverse effect by 
their effects on blood coagulation, notably on fibrinogen, factor VIIc and antithrombin III. ${ }^{22}$

It is currently unclear whether opposed oestrogen/ progestogen preparations are protective against cardiovascular disease, and with some preparations the risk of thromboembolism may actually be increased. ${ }^{20}$ This observation leads to a management dilemma, particularly regarding unopposed oestrogens. Patients with RVO have been reported to have an excess mortality from cardiovascular disease, supporting the idea that the protective effect of oestrogens in HRT would be beneficial. Equally, if HRT is implicated in the aetiology of RVO, then, should the therapy be stopped as in 1980s management? Evidence suggesting that HRT should be continued comes from a study of postmenopausal women who did not demonstrate a rise in blood pressure during prolonged (unopposed oestrogen) HRT $^{23}$ It would therefore appear that in view of its cardioprotective effect, HRT should be continued in patients who sustain RVO whilst taking this therapy.

\section{CONCLUSION}

This is the largest series of cases of RVO associated with sex hormone preparations. In our series, such patients make up the majority of young female patients sustaining RVO. This is obviously a matter of some concern, particularly as one of our patients had recurrence with poor visual outcome.

Regarding patients with RVO taking HRT, there appears to be no obvious relationship between RVO and HRT. With the large and increasing number of patients using HRT in this country and world-wide, this has wider implications. Hence, it is important to report such patients, particularly as the epidemiological effects of opposed HRT have yet to become fully apparent.

The mechanism for an increased risk of RVO with sex hormone preparations is unknown. Considering their effects on plasma lipoproteins and blood coagulability, with the prominence of the former as a risk factor for RVO in younger patients, both these detrimental effects of sex hormone preparations may have a significant role.

On the basis of the available (limited) data, it would seem prudent to stop the use of the oral contraceptive pill in patients sustaining retinal vein occlusion. However, our data do not suggest that use of hormone replacement therapy is associated with any excess risk of retinal vein occlusion.

Key words: Retinal vein occlusion, Oral contraceptive, adverse effects, Hormone replacement therapy, Oestrogen replacement.

\section{REFERENCES}

1. Sorsby A. The incidence and causes of blindness in England and Wales. London: HMSO, 1978:1963-8.
2. Klein BA. Occlusion of the central retinal vein: clinical importance of certain histo-pathologic observations. Am J Ophthalmol 1953;36:316-24.

3. Rabinowicz IM, Litman S, Michaelson IC. Branch venous thrombosis: a pathological report. Trans Ophthalmol Soc UK 1968;88:191-210.

4. Dodson PM, Kritzinger EE. Underlying medical conditions in young patients and ethnic differences in retinal vein occlusion. Trans Ophthalmol Soc UK 1985; 104:114-9.

5. Vessey MP. Female hormones and vascular disease: an epidemiological overview. Br J Fam Plann 1980;6:1-12.

6. Helmrich SP, Rosenberg L. Kaufman DW, Strom B, Shapiro S. Venous thromboembolism in relation to oral contraceptive use. Obstet Gynecol 1987;69:91-5.

7. Vessey MP, Lawless M, Yeates D. Oral contraceptives and stroke: findings in a large prospective study. BMJ 1984;289:530-1.

8. La Veccia C. Sex hormones and cardiovascular risk. Hum Reprod 1992;7:162-7.

9. Grimes DA. The safety of oral contraceptives: epidemiologic insights from the first 30 years. Am J Obstet Gynecol 1992;166:1950-4.

10. Thorogood M, Villard-Macintosh L. Combined oral contraceptives: risks and benefits. Br Med Bull 1993; 49:124-39.

11. Foster K, Jackson B, Thomas M, Hunter P, Bennett N. Contraception. In: General household survey. London: HMSO, 1993:113-36.

12. Godsland IF, Crook D, Wynn V. Clinical and metabolic considerations of long term oral contraceptive use. Am J Obstet Gynecol 1992;166:1955-63.

13. Fong ACO, Schatz H. Central retinal vein occlusion in young adults. Surv Ophthalmol 1993;37:393-417.

14. Cecily C, Kelleher MD. Clinical aspects of the relationship between oral contraceptives and abnormalities of the coagulation system: relation to the development of cardiovascular disease. Am J Obstet Gynecol 1990; $163: 392-5$

15. Bonnar J. Coagulation effects of oral contraception. Am J Obstet Gynecol 1987;157:1042-8.

16. Prentice RL. On the ability of blood pressure effects to explain the relation between oral contraceptives and cardiovascular disease. Am J Epidemiol 1988;127: 213-9.

17. Wynn V, Adams PW, Godsland I, et al. Comparison of effects of different combined oral-contraceptives on carbohydrate and lipid metabolism. Lancet 1979; 1:1049-54.

18. Fotherby K. Update on lipid metabolism and oral contraception. Br J Fam Plann 1990;15:23-6.

19. Lobo RA, Speroff L. International consensus conference on postmenopausal hormone therapy and the cardiovascular system. Fertil Steril 1994;61:592-5.

20. Meade TW, Berra A. Hormone replacement therapy and cardiovascular disease. Br Med Bull 1992;48: 276-308.

21. Lobo RA. The role of progestins in hormone replacement therapy. Am J Obstet Gynecol 1992;166: 1997-2003.

22. Meade TW, Stanwell-Smith R. Hormone replacement therapy for menopausal women: a review of its effects on homeostatic function, lipids, and blood pressure. Adv Drug Reac Rev 1984:4:187-210.

23. Lip GY, Beevers M, Churchill D, Beevers DG. Hormone replacement therapy and blood pressure in hypertensive women. J Hum Hypertens 1994;8:491-4. 